\title{
96 dB Linear High Dynamic Range CAOS Spectrometer Demonstration
}

\author{
Mohsin A. Mazhar and Nabeel A. Riza, Fellow, IEEE
}

\begin{abstract}
For the first time, a CAOS (i.e., Coded Access Optical Sensor) spectrometer is demonstrated. The design implemented uses a reflective diffraction grating and a timefrequency CAOS mode operations Digital Micromirror Device (DMD) in combination with a large area point photo-detector to enable highly programmable linear High Dynamic Range (HDR) spectrometry. Experiments are conducted with a $2850 \mathrm{~K}$ color temperature light bulb source and visible band color bandpass and high-pass filters as well as neutral density (ND) attenuation filters. A $\sim 369 \mathrm{~nm}$ to $\sim 715 \mathrm{~nm}$ input light source spectrum is measured with a designed $\sim 1 \mathrm{~nm}$ spectral resolution. Using the optical filters and different CAOS modes, namely, Code Division Multiple Access (CDMA), Frequency Modulation (FM)-CDMA and FMTime Division Multiple Access (TDMA) modes, measured are improving spectrometer linear dynamic ranges of $28 \mathrm{~dB}, 50 \mathrm{~dB}$, and 96.2 dB, respectively. Applications for the linear HDR CAOS spectrometer includes materials inspection in biomedicine, foods, forensics, and pharmaceuticals.
\end{abstract}

Index Terms - Spectrometer, Digital Micromirror Device, Spectroscopy, Optical MEMS, High Dynamic Range.

\section{INTRODUCTION}

$\mathrm{O}$ PTICAL spectrometry is a powerful measurement tool for numerous applications. Spectrometers can be designed for single spatial point/zone inspection or for pixel-by-pixel image format spectroscopic mappings [1]. There are a variety of methodologies to extract spectral information. Given the maturity of silicon-based image sensors such as CCD and CMOS image sensors, their use as sensors in spectrometer has been dominant for UV, visible and Near Infrared (NIR) spectrometry between $350 \mathrm{~nm}$ and $1100 \mathrm{~nm}$ [2-6]. For wavelengths exceeding $1100 \mathrm{~nm}$, other non-silicon [7] optical image photo-detectors (PD) technologies have been deployed at the expense of higher cost, limited dynamic range (DR) and greater nonlinearities. One solution to reduce costs and improve performance is the classic dispersive grating spectrometer design that uses a point PD and deploys a scanning slit or grating to time sequentially scan through the spectrum under measurement. A potential weakness of such a design is that only a small fraction of the light in the full spectrum is selected for photo-detection at any instant causing limitations in measured spectral bin Signal-to-Noise Ratio (SNR) leading to non-robust

Manuscript received by IEEE PTL J. August 31, 2020; accepted. Date of publication; date of current version. The authors are with Photonic Information readings.

Over 70 years ago, an elegant solution to solve this low SNR problem was proposed that involved using many simultaneous spatially coded slits using patterned disks allowing all spectral bins (i.e., much more total light power) to be detected at the same time giving higher SNR photo-detection and hence a higher reliability spectral measurement [8]. This technique was again independently proposed and developed over the following decades by other researchers and eventually was called Hadamard Transform (HT) spectrometry given that the Hadamard orthogonal matrix content was used for both spatially encoding the spectral channels and then decoding the spectral bins via inverse HT [9-11]. Interestingly spinning digital spatial coding optical disks were also proposed and demonstrated for RF spectrum analysis [12]. Another remarkable innovation happened on the optical spatial light modulation device front when in the late 1990's, the TI DMD became available for use by researchers [13]. The DMD is a highly reliable digital on/off micromirror tilt states near 2 million pixels (micromirrors) broadband (350-2700 nm) spatial light modulator with frame rates currently reaching $50 \mathrm{KHz}$. Researchers realized the value of the DMD and proposed and demonstrated systems involving optical spectral control for a host of applications [14-19]. In fact, the DMD was also deployed to realize a HT spectrometer [20] and even recently studies continue on the HT DMD-based spectrometer and its higher speed compressive designs [21-24].

Use of speckle via an inserted scattering medium has been recently suggested to improve the spectral range DR of a dispersion prism spectrometer that uses a CCD sensor camera as the image sensor PD, thus restricting wider bandwidth spectral usage [25]. It would also be highly desirable that a dispersive-optic based point PD spectrometer not only has a programmable adequate SNR, but also delivers spectral measurements that are linear and with a detected signal HDR (> $90 \mathrm{~dB}$ ). In addition, faster speeds than the classic HT spectrometer would be desirable. Recently the CAOS platform has been proposed and demonstrated [26] that allows the design of a SNR controllable linear extreme DR optical imager using

Processing Systems Laboratory, School of Engineering, University College Cork, Ireland. e-mail: n.riza@ucc.ie. 
the principles of the HDR multiaccess RF wireless phone network. CAOS exploits time-frequency coding of selected optical pixels along with low noise programmable gain coherent signal processing involving RF correlation and spectral processing to extract HDR optical pixel data. In addition, it has been pointed out that the CAOS platform when using its CDMA-mode [27] also allows the design of a faster speed spectrometer when compared to the HT spectrometer [28]. Hence, this letter presents the first demonstration of a linear HDR CAOS spectrometer. Specifically, a broadband light source is engaged with a DMD-based CAOS point PD grating spectrometer design to demonstrate linear HDR spectroscopy using ND optical filter calibrated color filterbased spectroscopic test targets. Described are the details of the CAOS spectrometer design, experiment and sample test results.

\section{CAOS SPECTROMETER}

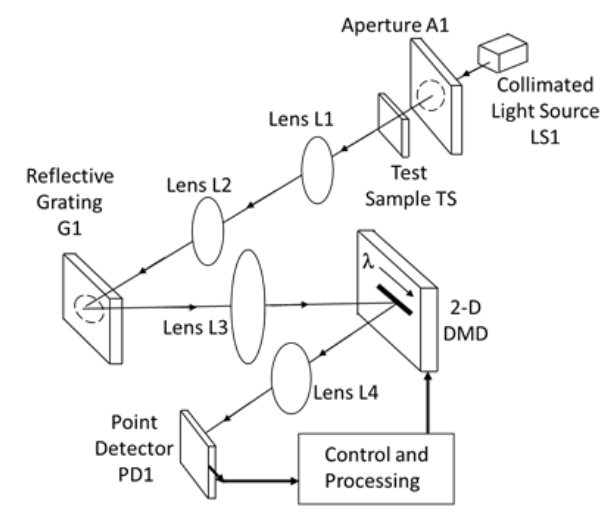

Fig. 13 -D view of the linear HDR CAOS spectrometer.

Fig.1 shows the 3-D view of a basic CAOS spectrometer design. A collimated beam from an external light source LS1 passes through iris aperture A1 and test sample TS to enter a telescopic lens system comprised of spherical lenses L1 and L2 with focal lengths $F 1$ and F2, respectively. The aperture size is controlled to illuminate the reflective grating G1 with the appropriate spot beam size given a larger beam size improves the system optical efficiency and diffraction limited spectral resolution while a smaller beam size improves the collimated beam illumination zone given the light source provides a partial spatially collimated beam. The surface normal of the grating makes an angle $\alpha$ with the beam optical axis and is adjusted for efficient placement of the desired spectrum on the DMD surface. After passing through the focus lens L3 with focal length F3, the spatially dispersed light from the grating representing the incident light spectrum forms a line illumination along the DMD horizontal axis labelled as $\lambda$. The +1 (i.e., $+\theta$ ) tilt micro-mirror state of the DMD sends light from the DMD plane through the imaging lens L4 of focal length F4 to be imaged on to a large area high speed point detector PD1. The photo-detected signal is amplified and sampled by a high speed Analog-to-Digital Converter (ADC) and then sent for time-frequency (Hz domain) Digital Signal Processing (DSP) operations via the control and signal processing electronics. Depending on the required linear DR needs of the spectral testing scenario, the CAOS spectrometer hardware is programmed to operate with the optimal time-frequency CAOS mode [27].

The basic CAOS-mode is the CDMA mode where each CAOS pixel chosen in the DMD micromirrors zone is assigned a unique binary code sequence in time selected ideally from an orthogonal binary code set such as from a Walsh code set. The location of these M CAOS pixels can be anywhere in the DMD optical spectral 1-D $\lambda$ grid space and the size and shape of the CAOS pixel in micromirror units is also programmable based on spectral resolution needs for the spectroscopic sample under test. In effect, the shortest time code sequences can be used in the CDMA-mode as only the few select CAOS pixels of spectral interest are harvested by the CAOS spectrometer making a higher speed spectrometer versus for example the HT spectrometer that implements continuous spatial coded space HTs [28]. Given all CAOS pixels of interest are photo-detected at the same time, higher light levels for photo-detection can lead to adequate SNR (i.e., >1) and adequate (e.g., $30 \mathrm{~dB}$ ) dynamic range spectral bin measurements. For CDMA-mode operations, light source power levels as well as photo-detector optical gain and electronic amplifier gain and filter settings in electronic hardware can be adjusted for optimal SNR spectral bin measurements. To extract spectral data over a higher linear DR, the FM-CDMA CAOS-mode can be deployed that not only provides a higher DR but also software-based SNR control via DSP gain adjustments. Specially, each CDMA code bit at a bit rate of $f_{B}$ bits/sec in the CAOS pixel time code sequence is modulated by an FM carrier at $f_{c} H z$ where $f_{C}=f_{B} P$ and $P$ is an integer. Recovery of the irradiance for a CAOS optical spectral bin is achieved in two decoding steps where the first step involves RF spectral processing of the FM carrier within each bit time via the DSP Fast Fourier Transform (FFT) algorithm to recover the irradiance of all the selected CAOS spectral bins and the second step involves decoding of these various simultaneous spectral bins that were encoded with CDMA codes. By controlling the $A D C f_{S}$ sampling rate, $f_{c}$ and $f_{B}$, the FFT DSP gain of $10 \log (\mathrm{N} / 2) \mathrm{dB}$ is controlled by controlling the number of data samples $\mathrm{N}$ for the N-point FFT processing. For lower noise and higher DR spectral bin recovery, both the CAOS spectral bins $\mathrm{M}$ and the sample count $\mathrm{N}$ per bit time can be optimized based on prior knowledge of sample spectral test data. To enable extreme linear DR, the FM-TDMA CAOS mode can be engaged where a single CAOS pixel is extracted per TDMA time slot, allowing optimal use of the full saturation capacity of the point PD. Previous data measured for a CAOS camera indeed highlighted these controllable SNR and linear DR features of the various CAOS modes and in fact have shown a $177 \mathrm{~dB}$ linear extreme DR capability for the imaging 
application [29]. The next section highlights similar linear HDR capabilities for the CAOS spectrometer instrument.

\section{EXPERIMENTS AND DISCUSSION}

The Fig.1 CAOS spectrometer is built in the laboratory using the following components. LS1: Avantes (UK) AvaLIGHTHAL-S-Mini Pro-lite $2850 \mathrm{~K}$ bulb color temperature, $4.5 \mathrm{~mW}$ power, $360-2500 \mathrm{~nm}$ spectrum, 600 micron diameter fiber feed with a $5 \mathrm{~cm}$ focal length $2.5 \mathrm{~cm}$ diameter collimation lens with a 2.1 degree output beam divergence; Vialux (Germany) DMD model V-7001 with micromirror size of $13.68 \mu \mathrm{m} \times 13.68$ $\mu \mathrm{m}$; DELL 5480 Latitude laptop for control and DSP, National Instruments 16-bit ADC model 6211; Thorlabs components that include test sample $450 \mathrm{~nm}$ and $620 \mathrm{~nm}$ color $10 \mathrm{~nm}$ FWHM bandpass filters FB620-10 and FB450-10 and a red dichroic band high-pass filter FD1R and a set of ND filters, point PMT Model PMM02 (and optional silicon point PD model PDA100A2 with $70 \mathrm{~dB}$ variable electronic gain amplifier), $4 \mathrm{~mm}$ diameter Iris A1, $5.08 \mathrm{~cm}$ diameter uncoated broadband lenses $\mathrm{L} 1 / \mathrm{L} 2 / \mathrm{L} 3$ with $\mathrm{F} 1=5 \mathrm{~cm}, \mathrm{~F} 2=\mathrm{F} 3=6 \mathrm{~cm}$, and a spherical mirror SM1 with $\mathrm{F} 4=3.81 \mathrm{~cm}$ focal length used instead of the lens $\mathrm{L} 4, \mathrm{G} 1$ with $\mathrm{f}_{\mathrm{G}}=600$ lines $/ \mathrm{mm}$ and $\mathrm{D}_{\mathrm{g}}=1.62$ $\mathrm{nm} / \mathrm{mrad}$ dispersion at $750 \mathrm{~nm}$. Key inter-component distances are: $11 \mathrm{~cm}$ between $\mathrm{L} 1$ and $\mathrm{L} 2$ giving an on-axis $6 \mathrm{~mm}$ diameter beam at the G1 plane with G1 rotated with incidence angle $\alpha=$ 6 degrees. $21.2 \mathrm{~cm}$ between $\mathrm{A} 1$ and G1, $10 \mathrm{~cm}$ between DMD and SM1/L4 and $6.2 \mathrm{~cm}$ between SM1/L4 and point PD1.

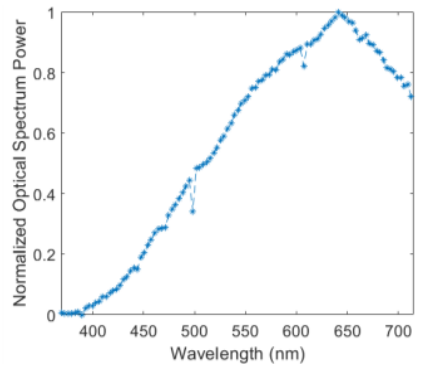

Fig. 2 Spectrometer full spectral system response via CAOS CDMA-mode.

Fig. 2 shows the experimental CAOS spectrometer system optical spectrum measured by the CAOS CDMA-mode using $\mathrm{W}=128$ bits length Walsh codes at a $\mathrm{f}_{\mathrm{B}}=1 \mathrm{KHz}$ bit rate and without placing a TS. A spectrum of $\sim 369 \mathrm{~nm}$ to $\sim 715 \mathrm{~nm}$ is observed over the full width of the DMD using M=102 CAOS pixels $(3.4 \mathrm{~nm} / \mathrm{CAOS}$ pixel) or $\sim 0.34 \mathrm{~nm} /$ micromirror as each CAOS pixel has a width of 10 micromirrors and a height of 300 micromirrors. The spectrometer wavelength axis is coarsely calibrated by placing known wavelength narrow $10 \mathrm{~nm}$ FWHM bandpass color filters as TSs such as at $450 \mathrm{~nm}, 550 \mathrm{~nm}$ and $620 \mathrm{~nm}$. Note that a CAOS pixel width of 3 micromirrors also allowed a full spectrum recovery indicating a system spectral resolution of $0.34 \times 3=1.02 \mathrm{~nm}$. Ideally, a fine electronically tuned calibrated optical filter should be used to calibrate the CAOS spectrometer wavelength axis on the DMD. Using highpass filter TS, Fig.3 shows the measured spectrum after normalization using the inverse of the Fig. 2 measured nonuniform system spectrum. At $550 \mathrm{~nm}$, the deployed high-pass filter has a designed filter spectral gain of zero, as also closely confirmed by the Fig. 3 spectral data at the $552.8 \mathrm{~nm}$ data point. This filter also has a rippled variable gain design near its rising band edge and this behavior is also confirmed in the Fig.3 data with a first maximum at $607.1 \mathrm{~nm}$. Note that other maximum response points also occur, including at the $631 \mathrm{~nm}$ data point.

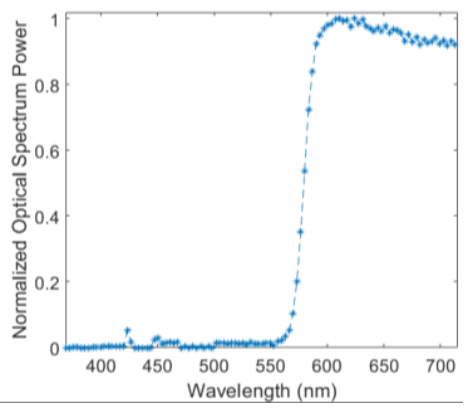

Fig. 3 Red color high-pass filter TS spectrum via CAOS CDMA-mode.

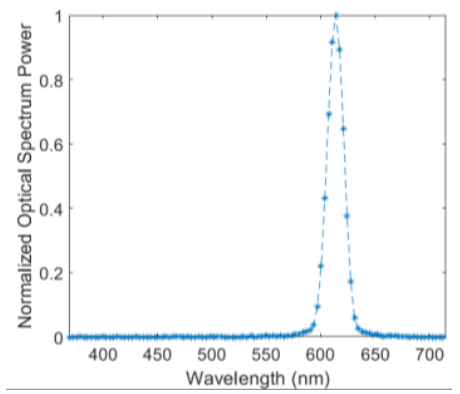

Fig.4 $620 \mathrm{~nm}$ bandpass filter at $\mathrm{OD}=2.5 \mathrm{TS}$ spectrum via FM-CDMA-mode.

The key feature provided by the CAOS spectrometer is programmable HDR and to test this aspect, the known $620 \mathrm{~nm}$ bandpass filter is used as a TS along with ND filters to attenuate the spectrum. Experiments show a robust test spectrum recovery for DR values of $28 \mathrm{~dB}$ and $50 \mathrm{~dB}$ for CDMA and FMCDMA modes, respectively. Fig. 4 shows the measured spectrum at the ND optical density (OD) filter value of 2.5 (i.e., $50 \mathrm{~dB}$ attenuation in the DR range) for the FM-CDMA mode using an $\mathrm{FM}$ of $\mathrm{f}_{\mathrm{c}}=520 \mathrm{~Hz}, \mathrm{~W}=128$ bits CDMA Walsh codes (via a $128 \times 128$ Sylvester construction Hadamard matrix) at $f_{B}=0.5 \mathrm{~Hz}$ bit rate $\left(2 \mathrm{sec}\right.$ bit time) giving $P=\mathrm{f}_{\mathrm{c}} / \mathrm{f}_{\mathrm{B}}=1040$ and an ADC sampling rate of 65535 Sps giving $\mathrm{N}=131072$ for N-point FFT gain of $48 \mathrm{~dB}$. Fig. 4 shows the measured spectrum using the $620 \mathrm{~nm}$ bandpass filter TS with a measured $16.3 \mathrm{~nm}$ FWHM versus the ideal $10 \mathrm{~nm}$ given the non-optimal optical design of the system that does not minimize and compensate image blur and optical aberrations such as by custom techniques [30]. In addition, the system is designed using paraxial (i.e., near onaxis) rays, but non-paraxial conditions apply as the LS1 beam is not ideally collimated and has a 2.1 degree divergence that gives a near $14 \mathrm{~mm}$ spread for the $\Delta \lambda=346 \mathrm{~nm}$ full spectrum light on the DMD versus a $\sim 12.5 \mathrm{~mm}$ width using the grating frequency-based spectral width paraxial limit design expression of $\mathrm{f}_{\mathrm{G}} \Delta \lambda \mathrm{F}$ where $\mathrm{F}=\mathrm{F} 3$ for focus lens L3.

To improve the spectrometer recovery DR beyond $50 \mathrm{~dB}$ DR, stronger attenuation ND filters are applied and the FM-TDMA 
mode is engaged for a larger CAOS pixel covering the null-tonull $620 \mathrm{~nm}$ spectrum with $\mathrm{FM} \mathrm{f}_{\mathrm{c}}=520 \mathrm{~Hz}$ and $\mathrm{ADC}$ sampling TDMA one time slot duration of $\mathrm{T}_{\mathrm{D}}=2 \mathrm{sec}$. Again a $48 \mathrm{~dB}$ FFT gain is used and robust spectral recovery is achieved up-to $\mathrm{OD}=4.8$ giving a $96.2 \mathrm{~dB}$ measured HDR with an $\mathrm{SNR}=1.2$. Fig.5 shows the high linearity plot of the $96.2 \mathrm{~dB}$ HDR response of the CAOS spectrometer during the $620 \mathrm{~nm}$ filter TS test. Note that experiments with the point silicon PD as PD1 also produced HDR spectral results from the CAOS spectrometer, e.g., $\mathrm{HDR}=80 \mathrm{~dB}$ using the CAOS FM-TDMA mode.

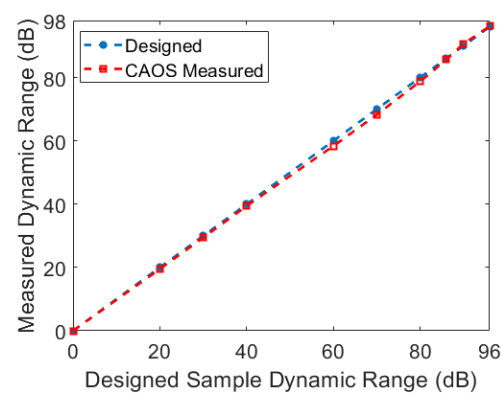

Fig. 5 Linearity plot for the $96 \mathrm{~dB}$ HDR response of the CAOS spectrometer.

To summarize the experimental results, first note that the reported wavelength range is only guaranteed when this spectrometer is compared with spectral measurements done with a commercial grade reference spectrometer. Also note that the spectrometry processing time is dominated by the deployed CAOS mode's encoding time $T_{E}$ that depends on the chosen CAOS-mode experimental parameters. For the Fig.2 \& 3 CDMA-mode data, $\mathrm{T}_{\mathrm{E}}=\mathrm{W} / \mathrm{f}_{\mathrm{B}}=128 /(1 \mathrm{KHz})=0.128 \mathrm{sec}$ while the Fig.4 FM-CDMA mode data uses a $\mathrm{T}_{\mathrm{E}}=\mathrm{W} / \mathrm{f}_{\mathrm{B}}=128 /(0.5 \mathrm{~Hz})=$ $256 \mathrm{sec}$. Using a fastest $\mathrm{f}_{\mathrm{c}}=25 \mathrm{KHz}, \mathrm{T}_{\mathrm{E}}$ can be reduced to 5.33 sec. The Fig.5 FM-TDMA mode data uses a $\mathrm{T}_{\mathrm{E}}=\mathrm{T}_{\mathrm{D}}=2 \mathrm{sec}$ for each CAOS spectral reading. The highest linear HDR measured for the spectrometer is $96 \mathrm{~dB}$ with a minimum optical signal detected power of $10.1 \mathrm{pW}$ maintaining a $\mathrm{SNR} \geq 1.2$.

\section{CONCLUSION}

For the first time, the CAOS spectrometer is demonstrated. Experiments using known spectral response color and ND filter test samples highlight the robust highly programmable spectral recovery. Specifically, spectrum measurements by the CAOS spectrometer with increasing test sample DR values from 28 $\mathrm{dB}, 50 \mathrm{~dB}$, and $96.2 \mathrm{~dB}$ are achieved using CAOS CDMA, FMCDMA, and FM-TDMA modes, respectively. The proposed CAOS spectrometer can be optimized for a chosen application and spectral range, include IR bands exceeding $1100 \mathrm{~nm}$ where non-silicon (e.g., Germanium) point PDs can be deployed.

\section{REFERENCES}

[1] R. A. Crocombe, "Portable spectroscopy," Applied Spectroscopy , 72(12), 1701-1751, 2018.

[2] J. Chen, F. Cai, F., R. He, \& S. He, "Experimental demonstration of remote and compact imaging spectrometer based on mobile devices," MDPI Sensors J., 18(7), 1989, 2018.

[3] L. Hong, Lingyu, and K. Sengupta, "Fully integrated optical spectrometer in visible and near-IR in CMOS," IEEE Transactions on Biomedical Circuits and Systems, 11, no. 6, 1176-1191, 2017.

[4] M. A. Hossain, J. Canning, K. Cook \& A. Jamalipour, "Optical fiber smartphone spectrometer," Optics Letters, 41(10), 2237-2240, 2016.

[5] E. V. Woodburn, K. D. Long, and B. T. Cunningham, "Analysis of paper-based colorimetric assays with a smartphone spectrometer," IEEE Sensors Journal 19, no. 2, 508-514, 2018.

[6] C. Zhang, G. Cheng, P. Edwards, M. D. Zhou, S. Zheng \& Z. Liu, "GFresnel smartphone spectrometer," Lab on a Chip, 16(2), 246-250, 2016.

[7] T. Iwata, K. Kitazato, M. Abe, M. Ohtake, T. Arai, C. Honda, N. Imae \& M. Komatsu, "NIRS3: the near infrared spectrometer on Hayabusa2," Space Science Reviews, 208(1-4), 317-337, 2017.

[8] M. J. E. Golay, "Multi-slit spectrometry," J. Opt. Soc. Am. 39(6), 437444, 1949.

[9] R. N. Ibbett, D. Aspinall, and J. F. Grainger, "Real-Time Multiplexing of Dispersed Spectra in Any Wavelength Region," Applied Optics, Vol. 7, No. 6 , pp. 1089-1093, June 1968.

[10] J. A. Decker, Jr. and M. 0. Harwitt, "Sequential Encoding with Multislit Spectrometers," Applied Optics, Vol. 7, No. 11 , pp. 2205-2209, November 1968.

[11] J. A. Decker, Jr., "Experimental realization of the multiplex advantage with a Hadamard-transform spectrometer," Applied Optics, 10(3), 510514, 1971.

[12] N. A. Riza, Chapter 4: Optical Disk-based Acousto-Optic Spectrum Analysis, Caltech Ph.D. Thesis, Oct. 1989.

[13] M.R. Douglass, "Lifetime Estimates and Unique Failure Mechanisms of the Digital Micromirror Device," IEEE International Reliability Physics Symposium, 36th Annual, pp. 9-16, April 1998.

[14] K. Kearney, and Z. Ninkov, "Characterization of a digital micro-mirror device for use as an optical mask in imaging and spectroscopy," Proc. SPIE 3292, 81, 1998.

[15] N. A. Riza \& S. Sumriddetchkajorn, "Digitally controlled fault-tolerant multiwavelength programmable fiber-optic attenuator using a twodimensional digital micromirror device," Opt. Lett., 24(5), 282-284, 1999.

[16] N. A. Riza and M. J. Mughal, "Broadband optical equalizer using faulttolerant digital micromirrors," Optics Exp., 11(13), pp.1559-1565, 2003.

[17] C. H. Chuang \& Y. L. Lo, "Digital programmable light spectrum synthesis system using a digital micromirror device," Applied Optics, 45(32), 8308-8314, 2006.

[18] D. L. Graff \& S. P. Love, "Toward real-time spectral imaging for chemical detection with a digital micromirror device-based programmable spectral filter" Journal of Micro/Nanolithography, MEMS, and MOEMS, 13(1), 011111, 2013.

[19] A. Elahi, and M. Shahabadi, "A grating-optic-less visible spectrometer using Fresnel zone plate patterns on a digital light processor," IEEE Sensors Journal, 18(15), pp.6432-6437, 2018.

[20] R. A. DeVerse, R. M. Hammaker, and W. G. Fateley, "Realization of the Hadamard multiplex advantage using a programmable optical mask in a dispersive flat-field near-infrared spectrometer," Applied Spectroscopy 54.12: 1751-1758, 2000.

[21] J. L. Xu, H. Liu, C. B. Lin \& Q. Sun, “ SNR analysis and Hadamard mask modification of DMD Hadamard Transform Near-Infrared spectrometer," Optics Communications, 383, 250-254, 2017.

[22] J. Kang, X. Li, \& Q. Liu, "Hadamard transform-based calibration method for programmable optical filters based on digital micro-mirror device," Optics Express, 26(15), 19563-19573, 2018.

[23] N. A. Riza, "A method and system for performing high speed optical image detection," Patent Appl. 19186121, 2019.

[24] Y. Zhang, J. Kang, C-M Hsieh, \& Q. Liu, "Compressive Optical Spectrometry Based on Sequency-Ordered Hadamard Transform," IEEE Photonics Journal, vol. 12, no. 5, pp. 1-8, Oct. 2020.

[25] Ş. K. Çetindağ, M. F. Toy, O. Ferhanoğlu and F. Çivitci, "A speckleenhanced prism spectrometer with high dynamic range," IEEE Photonics Technology Letters, 30, no. 24: 2139-2142, 2018.

[26] N. A. Riza, M. J. Amin, and, J. P. La Torre, "Coded Access Optical Sensor (CAOS) Imager," JEOS Rapid Publications, vol. 10, pp. 1502118, 2015. DOI: $10.2971 /$ jeos.2015.15021

[27] N. A. Riza and M. A. Mazhar, "Laser beam imaging via multiple mode operations of the extreme dynamic range CAOS camera," Applied Optics, vol. 57, no. 22, pp. E20-E31, June 2018.

[28] N. A. Riza \& M. A. Mazhar, "Pixels of Interest (POI) empowered higher speed CAOS camera imaging and spectrometry for multi-target applications," Optics Communications, 459, 124904, 2020.

[29] N. A. Riza \& M. A. Mazhar, "177 dB linear dynamic range pixels of interest DSLR CAOS camera,” IEEE Photonics J., 11(3), 1-10, 2019.

[30] B. K. Gunturk \& X. Li, Eds. Image restoration: Fundamentals \& Advances, CRC Press, 2012. 